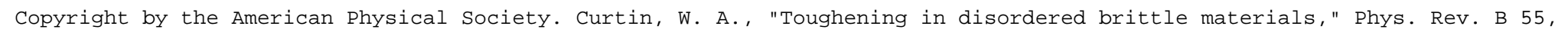
11270 DOI: http://dx.doi.org/10.1103/PhysRevB.55.11270

\title{
Toughening in disordered brittle materials
}

\author{
W. A. Curtin \\ Departments of Engineering Science \& Mechanics and Materials Science \& Engineering, Virginia Polytechnic Institute \\ and State University, Blacksburg, Virginia 24061
}

(Received 7 October 1996)

\begin{abstract}
The growth of a planar crack through a heterogeneous brittle material is investigated using a discrete cubic lattice of springs with distributed spring toughnesses and lattice Green's functions to determine crack propagation. The toughness, or stress required to grow an initial crack, is found to be a stochastic quantity and depends on the width of the distribution. For narrow distributions, the toughness is less than the thermodynamic value and is controlled by the nucleation of kinks at low toughness regions (weakest links), which then grow laterally in an unstable manner. For broad distributions, the average toughness approaches the thermodynamic value, with some specific configuration having greater values, and is controlled by high toughness regions pinning a rough crack front. The rough crack front exhibits nontrivial scaling with crack width and a "strongest-link"' behavior that differs from the usual weak-link behavior found in weakly disordered materials. Materials with broad distributions are also less sensitive to small preexisting defects. The difference in toughness between narrow and broad distributions is only about $10 \%$; that is much smaller than suggested by similar studies on $2 d$ materials and demonstrates the very important role played by geometry-dimensionality in this problem. One implication of these results is that toughness in complex or heterogeneous materials does not stem from simple disorder in toughnesses; more complex and microstructure-specific mechanisms such as microcracking and grain bridging must occur. [S0163-1829(97)01218-6]
\end{abstract}

\section{INTRODUCTION}

Most engineered materials and advanced composites have heterogeneous microstructures, and such disorder can strongly influence crack propagation and material failure. Standard analyses of crack propagation and failure, are, however, based on concepts strictly appropriate for homogeneous materials. The most celebrated of these analyses is the Griffith result for ideal brittle materials, which predicts that the unstable growth of a planar crack of length $2 c$ under perpendicular uniform tension initiates at the stress $\sigma=K_{I c} /(\pi c)^{1 / 2}$, where $K_{I c}$ is the material toughness. ${ }^{1}$ The toughness is an intrinsic material property, $K_{I c}=(\gamma E)^{1 / 2}$, with $E$ the Young's modulus and $\gamma$ the surface energy of the materials. The extension of the Griffith result to a heterogeneous material as simple as a polycrystalline ceramic with various crystallite orientations and/or grain boundary energies is not obvious. The Griffith result assumes self-similar crack growth, which does not necessarily occur in disordered materials, and the use of an average surface energy $\bar{\gamma}$ requires that all crack growth occur in a stable manner and so represents an upper, thermodynamic, bound on the average toughness over extended amounts of crack growth. Experimentally, it is well established that the toughness of many polycrystals, even in cubic ceramics which do not exhibit thermal-expansion anisotropy and "grain bridging" toughening, is larger than in single crystals. ${ }^{2}$ A related observation is the "trapping" of initially sub-grain-size cracks by the microstructure as the small cracks grow to sizes larger than the grain size of the material. While qualitative descriptions of the "trapping" have invoked local variations in fracture energy, ${ }^{2}$ and some work on pinning by tough inclusions has been carried out, ${ }^{3,4}$ the understanding of this phenomenon is incomplete.
Recent studies of the mechanics of disordered materials have focused on either effective continuum models or discrete numerical models. The discrete models utilize springs in a lattice; the springs are assigned various mechanical properties (stiffness, strength, thermal expansion) to create a disordered microstructure, and the stress-strain response is then simulated numerically. ${ }^{5,6}$ Interesting behavior has been found for materials with large disorder and no preexisting defects: failure occurs by the nucleation and growth of cracklike defects, with extensive nonlinear stress-strain behavior prior to failure. However, the mechanical properties of real brittle materials are often controlled by precisely the initial cracklike defects missing in the problems studied to date. The "ductile-like", behavior found in the highly disordered materials, which stems from extensive damage initiation and stable growth of fairly small cracks, may not persist in the presence of large initial defects. Most previous studies have also been on one- and two-dimensional (2D) systems, and geometry-dimensionality can play a crucial role in crack growth of heterogeneous systems. Toughness, or resistance of a material to growth of a large preexisting crack, and the influences of geometry and dimension have rarely been studied theoretically in heterogeneous materials. ${ }^{7,8}$ Sophisticated techniques for following planar crack growth in $3 d$ have been developed, but only toughness in ordered composite materials has been studied to date. ${ }^{4}$ The major issue studied here of fracture toughness in disordered materials has not been investigated previously despite its prime importance in nearly all applications of brittle materials.

Here, we specifically investigate the toughness of disordered three-dimensional brittle materials by explicitly measuring, via simulation, the stress required to grow a planar crack through a disordered material. We demonstrate that both the toughness and nature of crack growth depend on the 
extent of the disorder. For small or weak disorder, the crack front stays essentially planar and crack growth is initiated by the weakest regions along the crack front and is followed by unstable lateral crack growth. The toughness is then less than the thermodynamic average. For large or strong disorder the crack front evolves in a stable manner, and becomes rough as it is "pinned" by the tougher regions at the crack front. The rough crack front is caused by advance of the crack into the less-tough regions ahead of it, and this creates additional stress concentrations on the lagging pinning elements. However, for sufficiently large disorder, the tougher regions can withstand such stress concentrations, and the toughness approaches the thermodynamic value. The strongly disordered materials inhibit unstable crack growth, and also make the material resistant, or insensitive, to small-scale defects. The differences in average toughness between weak and strong disorder are fairly small, in contrast to the implications of previous $2 \mathrm{D}$ results. ${ }^{5,6}$ Our results clearly demonstrate the importance of geometry and dimensionality in determining resistance to crack growth and also indicate that disorder alone is not sufficient to generate substantial toughening. Real heterogeneous complex ceramics must be toughened by other specific mechanisms such as grain bridging and microcracking.

The remainder of this paper is organized as follows. In Sec. II, we describe the model brittle material used here and the computational aspects of simulating crack growth in these systems. In Sec. III, we present results for the toughness as a function of the disorder and lateral crack width, and discuss simple analytic models which quantitatively account for the behavior in the weak-disorder regime. Section IV contains further discussion, an investigation of the sensitivity of the disordered materials to initial defects, and further general implications of our findings.

\section{PLANAR CRACK GROWTH MODEL}

The physical problem of interest here is the growth of a crack through a heterogeneous brittle material, which might be a polycrystalline material having crystal orientationdependent fracture energies, a polycrystal with varying grain-boundary energies-toughnesses which fails transgranularly, or an intimate composite mixture of materials with varying interfacial energies. In each case, the heterogeneity is in both geometry and toughness-energy, and the characteristic length scale for variations in the disorder is the grain size of the material. To fully represent a 3D multiphase material with a complex crack shape is a daunting computational task, and some simplification of the microstructure is necessary. Here, we represent the heterogeneous material as a discrete cubic lattice of springs. Each spring represents a particular grain or grain boundary in the material and can be assigned properties (modulus, strength-toughness) closely corresponding to the actual material and its heterogeneity. Springs with zero modulus correspond to failed springs and are thus effectively cracks or parts of a larger crack. The mapping of the microstructure onto such a spring model generates a well-defined discrete mechanical system with a controllable "microstructure." A finer mesh of spring elements is desirable for more detail in the stress fields but becomes much less computationally efficient, and so we discretize on

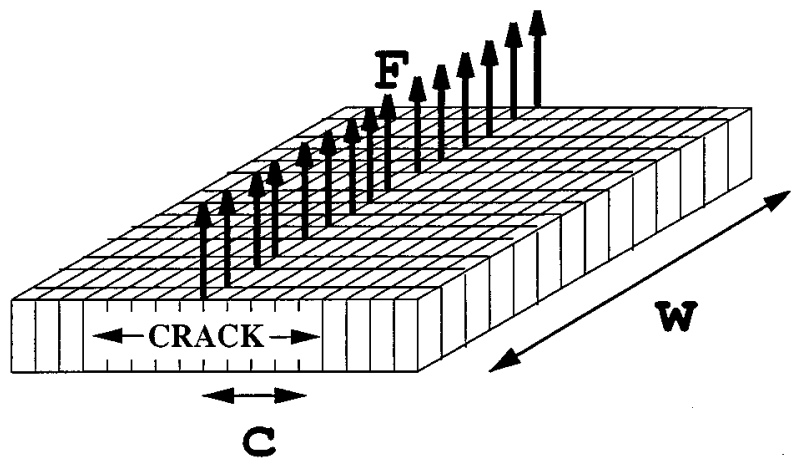

FIG. 1. Schematic of the cubic spring network in the plane of the crack.

the scale of the heterogeneity in the real materials as a first attempt at understanding the material behavior.

Since real planar cracks in nominally isotropic materials tend to remain planar and exhibit limited amounts of out-ofplane deflection (except during unstable fast fracture, which is not considered here) it is not unreasonable to neglect the geometric disorder in favor of the toughness disorder. Hence, here we consider planar crack growth only; slight geometric disorder can be partially accounted for by projecting its effects onto the crack plane. The restriction to planar crack growth is also necessary because we do not have the computational ability to allow the crack to wander out of the plane. However, the study of 2D planar crack growth in a 3D elastic medium, is a major advance over nearly all previous studies on 2D systems with $1 \mathrm{D}$ cracks.

The model brittle material studied here is thus a cubic lattice of elastic springs which is infinite in the $y$ direction (the axis of tensile loading), infinite in the $x$ direction (the direction of crack growth) with reflection symmetry about $x=0$, and periodic in the $z$ direction (the width $L$ of the crack). The initial cubic spring lattice consists of two types of springs connecting the various nodes, with nodes labeled by their $x, y$, and $z$ coordinates $l, m$, and $n$, respectively, and node displacements labeled by $u_{l, m, n}$. Springs of tensile stiffness $E$ connect nodes along the $y$ axis, i.e., the force along the $y$ axis in the spring connected to nodes $l, m, n$ and $l, m+1, n$ is $E\left(u_{l, m+1, n}-u_{l, m, n}\right)$. Pure shear springs of stiffness $\mu$ connect neighboring nodes in the $x-z$ plane so that, for instance, the force along the $y$ axis associated with nodes $l, m, n$ and $l+1, m, n$ is $\mu\left(u_{l+1, m, n}-u_{l, m, n}\right)$. Node displacements are confined to the $y$ direction only for simplicity. The general elastic model described above is thus recognized as the Rosenstock-Newell model introduced several decades ago. ${ }^{9}$ Here we use $E=\mu$, which can be modified if desired.

To measure toughness, an initial planar crack of length $\pm c$ around $x=0$ and spanning the width $L$ is first inserted into the $y=0$ plane by setting the spring moduli to zero in the first $c$ "rows" (strips of $L$ springs perpendicular to the $x$ axis, see Fig. 1) of springs. The remaining springs in the $y=0$ plane are assigned strengths $s$ (maximum supportable forces) randomly chosen from a preselected distribution $P(s)$. Tensile point forces $F_{\text {app }}$ are then applied across the nodes at $x=0$ only. For such a center loaded crack, the force $F$ at the crack tip $x=c$ is $F \propto F_{\text {app }} / c^{1 / 2}$ and decreases as the crack extends so that additional force must be applied to 
continue crack growth. Growth thus occurs in a stable manner, and this loading is analogous to the double cantilever beam and compact tension test geometries used to measure toughness in real materials. For a given force $F_{\text {app }}$, the local forces $f_{i}$ on all of the unbroken springs in the $y=0$ plane ahead of the existing crack are calculated using a lattice Green's-function technique. ${ }^{10-12} \mathrm{We}$ then focus on the springs only along the crack front, defined as any intact springs that are near neighbors of broken springs. At the crack front, the local force $f_{i}$ on spring $i$ represents the intensity $K_{i}$ of the square-root singularity of the stress field that would exist in a continuum of the same shape around spring $i$. For springs not on the crack front, the forces do not represent a stress-intensity factor: there is no crack tip to provide the underlying physical origin for the divergence. For any spring along the crack front with $f_{i}>s_{i}$ the local strength is exceeded and the spring is then "broken" by changing that $E_{i}$ to zero, and the crack grows. Once any new springs have been broken at a given applied force, the local forces $f_{i}$ are recalculated for the new crack shape and further crack growth is considered. If no springs have $f_{i}>s_{i}$ then the crack exists in a stable mechanical equilibrium state. The applied force $F_{\text {app }}$ is then increased such that exactly one more spring along the crack front satisfies $f_{i}=s_{i}$, and that spring is then broken. By this algorithm, the evolution of the crack front shape versus applied force $F_{\text {app }}$ can be monitored.

The toughness is measured by the applied force $F_{\text {app }}$ required to advance the planar crack forward by one spring across the entire width of the crack. Thus, one complete "row," or width of initially intact material, has failed. This measure is consistent with the conceptual picture of toughness in which a strip of material well ahead of the crack is failed, removed from the far field, and then pasted onto the back of the crack, thereby advancing the crack forward by the width of the strip. In disordered materials, the diffuse crack front varies as the crack grows forward but stays roughly constant in length, so that the damage zone ahead of the crack is a "near-tip process zone" that does not interfere with the conceptual cutting and pasting picture of advancing the crack. To compare various disordered systems, we normalize the force $F_{\text {app }}$ required to extend the crack by the corresponding value $F_{\text {app }}^{0}$ needed in the homogeneous system (all local strengths $s_{i}=1$ ).

The alert reader will recognize that the crack growth, or spring breaking, criterion used here is a stress-force criterion. That is, local failure occurs when a spring force reaches some preassigned critical value. Fracture should be determined as the point at which the elastic energy that is released when the spring breaks equals the surface energy $\gamma$ required to break the spring. This is the fundamental thermodynamic concept of fracture introduced by Griffith. Only years later was it shown that this fracture condition corresponds precisely to one based on a critical stress intensity factor $K_{i c}$ $=(\gamma E)^{1 / 2}$ so that stress intensity can be used as a fracture criterion in homogeneous systems. Stress-based criteria are rarely used in theoretical mechanics and should be avoided, although to date such a criterion has been used almost exclusively in spring network models of fracture with no a priori justification. ${ }^{5,6}$ In the present discrete elastic system, however, the "strain energy release rate" $G$, or decrease of elastic energy upon failure of a spring, turns out to be essentially directly related to the force on the spring at the breaking point. Specifically, for a number a various crack front geometries, we have measured the released potential energy $G$ after spring breaking and find that $G=1.54 f_{i}^{2}$ where $f_{i}$ is the force on the spring $i$ prior to breaking. The factor of 1.54 is accurate to within $\pm 1 \%$ for a wide range of crack geometries. Hence, although we have assigned a local strength $s_{i}$ to each spring, this is equivalent to assigning a (dimensionless) local surface energy $\gamma_{i}=1.54 s_{i}{ }^{2}$ to that same spring. Taking fracture to occur when the local force $f_{i}$ reaches the value $s_{i}$ is therefore equivalent to taking fracture to occur when the energy release rate $G$ reaches the local surface energy $\gamma_{i}$. For our particular model, then, we have an explicit relationship between a local force condition for failure and an energy condition for failure. Below, we consider distributions of spring strengths described by some $P(s)$. As $P(s)$ is changed to maintain a fixed mean value of $s$, the mean value of the surface energy does change because it is the second moment of the strength distribution. The appropriate average (dimensionless) toughness for a given $P(s)$ is thus the square root of the average surface energy,

$$
\bar{K}=\left(\int s^{2} P(s) d s\right)^{1 / 2} .
$$

This value is the "thermodynamic" fracture toughness to which our results must be compared.

\section{TOUGHNESS VERSUS DISORDER}

\section{A. Results}

We apply the modeling approach described above to study crack propagation through materials with toughness distributions of the form

$$
\begin{aligned}
P(s) & =\frac{1}{W}, & 1-\frac{W}{2}<s<1+\frac{W}{2}, \\
& =0 & \text { otherwise }
\end{aligned}
$$

such that the distribution width is $W(0<W<2)$ and the mean strength is always unity. This is the same distribution used by Khang et al. to study the fracture of 2D square lattices in the absence of any initial defects. ${ }^{5}$ Khang et al. found that for small $W$ the material failed by unstable growth of a small nucleated crack whereas for $W=2$ the material could sustain a finite fraction of damage prior to failure. $W=2$ was then coined "ductile-like" and "tough" in the sense that the work of fracture (area under the stress-strain curve) was enhanced, although actual fracture toughness was not considered. Here, we consider the 3D case of the model studied by Khang et al. in the presence of the large initial crack, and can thus assess whether or not materials with broadly distributed disorder $(W=2)$ have enhanced fracture toughness. Since damage out of the crack plane is prohibited, however, our results are not a precise generalization of the Khang et al. results to 3D. To properly assess toughening, we must compare the measured failure forces to the (dimensionless) thermodynamic toughness of Eq. (1) which, for the distributions given by Eq. (2), is 


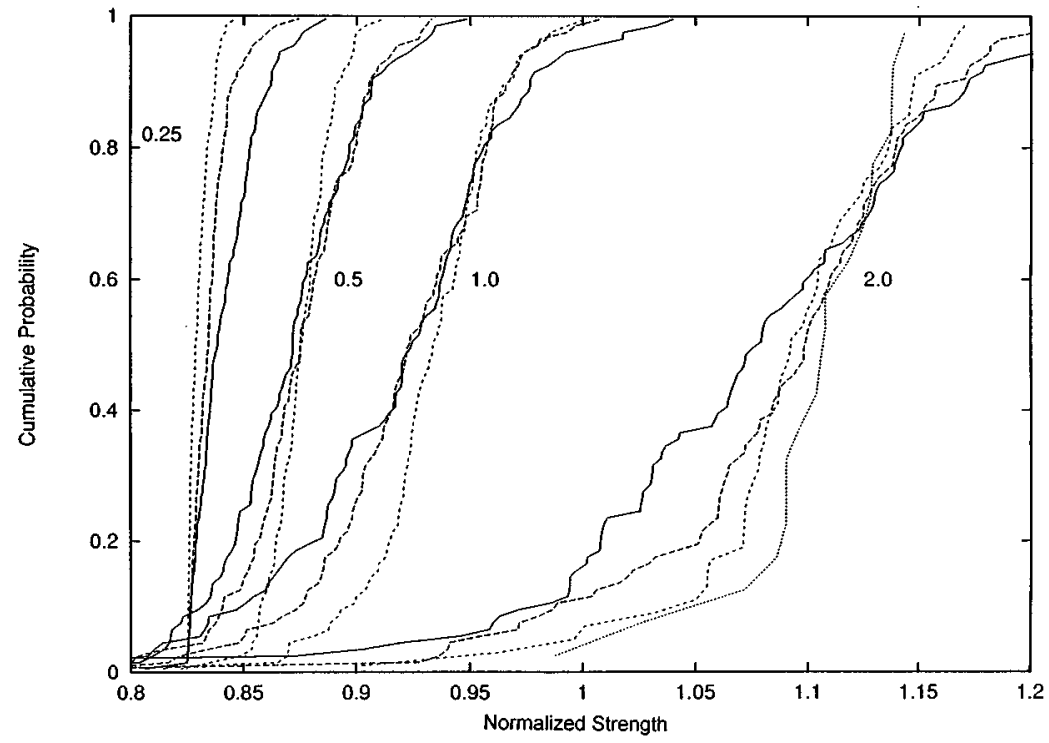

FIG. 2. Probability of failure vs normalized strength for various values of disorder variable $W$ and crack width $L(-, L=20 ;----, L=40$; ----, $L=100 ; \cdots, L=200)$. Note: the data for $W=0.25$ have been shifted down by -0.05 for clarity.

$$
\bar{K}=\left(1+\frac{W^{2}}{12}\right)^{1 / 2}
$$

We start with an initial crack size of $c=15$ (15 rows of initially broken springs), which is large enough that the results do not depend on $c$ (identical results with $c=8$ and 40 have been obtained). Each increment of crack growth is a single measure of the toughness that depends on the precise configuration of local toughnesses. So, many increments of crack growth must be studied and a statistical distribution of toughnesses must be considered. This is an unappreciated point: in a heterogeneous material the toughness itself is a statistical quantity and not a "material property" as in a homogeneous material. Results for the normalized force to grow the crack in various heterogeneous materials are shown in Fig. 2 as a cumulative probability distribution, for values of $W=0.25,0.5,1.0$, and 2.0, and for sample widths of $L$ $=20,40,100$, and, for $W=2.0$ only, 200. Evidently, the strength of weakly heterogeneous materials $(W<0.5)$ is rather lower than the average strength, and is very narrowly distributed around 0.88 . In contrast, the strength for the most heterogeneous material ( $W=2.0$ ) is greater than the average and more broadly distributed around 1.10 , with some con-

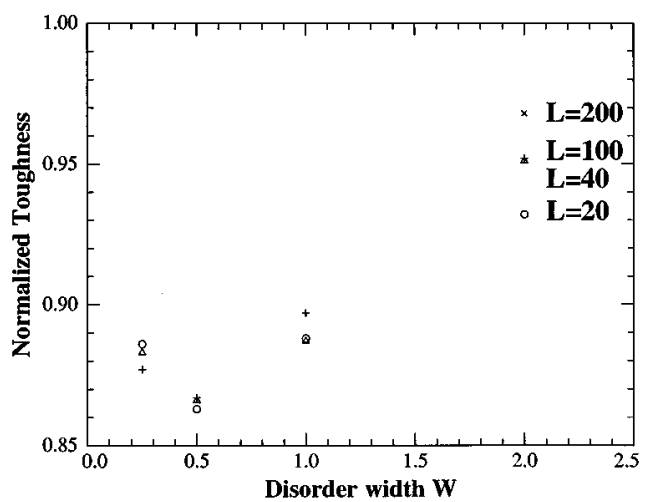

FIG. 3. Average normalized toughness vs disorder width $W$ for various crack widths $L$. figurations as strong as 1.20. The difference in strength between weak disorder and strong disorder is about $25 \%$.

Figure 3 shows the average strength results of Fig. 2 but now normalized by the thermodynamic toughness [Eq. (3)]. The weakly disordered materials still exhibit toughnesses below the thermodynamic toughness, while the strongly disordered materials $(W=2.0)$ show average toughnesses approaching, with increasing crack width $L$, the thermodynamic value. Some configurations for $W=2.0$ exhibit toughnesses exceeding the thermodynamic average value, which is physically allowed when clusters of tougher springs exist in the region ahead of the crack. Nonetheless, the difference in toughness between weak disorder and strong disorder is only about $10 \%$. This is a main result of our work.

Figure 4(a) shows the crack growth process for $W=0.25$. Growth occurs by single-kink formation just ahead of the crack at the very weakest heterogeneity followed by unstable lateral crack growth. The unstable lateral crack growth indicates that the thermodynamic average toughness will not be attained. Figure 4(b) shows a portion of the crack front for $W=2$ just after the crack advance: the crack front is diffuse and rough, and at some points extends 4 or 5 elements ahead of the initial crack line but is pinned at a few

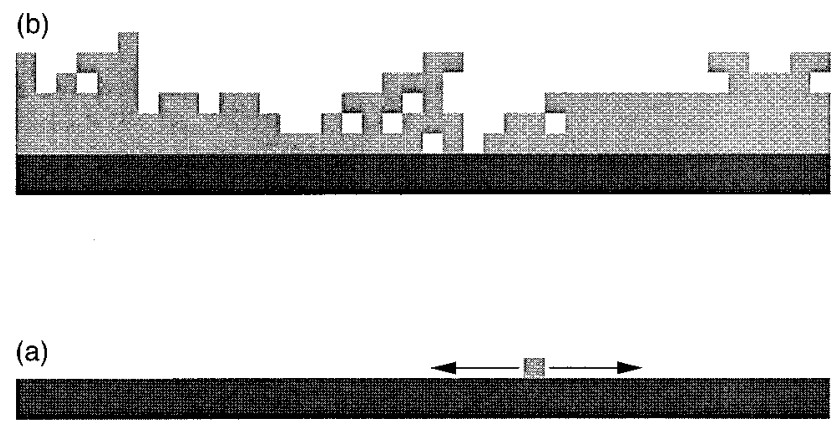

FIG. 4. Schematic of the crack advance sequence in two cases: (a) weak disorder $(W=0.25)$ in which kink nucleation leads to unstable lateral crack growth; (b) strong disorder $(W=2.0)$ where the crack front is diffuse and pinned by a few strong heterogeneities. 
key points by tough heterogeneities. The crack grows very stably by a failure of one, or just a few, springs at a time. Such stable crack growth drives the toughness toward the thermodynamic average. It should also be noted that in only two particular realizations was complete failure of row 16 (just ahead of the initial crack at row 15) actually preceded by complete failure in row 17; thus the pinning sites are essentially never disconnected from the crack front and no "bridges" are formed. The extent of damage growth ahead of the crack, as measured by the number of broken springs $N_{b}$ just after crack advance divided by the crack width $L$, is precisely zero for $W=0.25$ but for $W=2.0$ depends explicitly on the sample width $L$ as, roughly, $N_{b} / L \propto 0.55 L^{0.3}$ for $20<L<200$. This indicates that there is no intrinsic lateral scale for the fluctuation in the crack front, at least up to $L$ $=200$. The details of this apparent "roughening transition" with increasing disorder will be studied further in a future publication. Accompanying the transition from a flat to a rough crack interface is a narrowing of the distribution of toughnesses with increasing system size (see Fig. 2) which occurs by elimination of the low-strength tail of the distribution.

\section{B. Analytic analysis}

Here, we rationalize some of the results found in the simulations presented above through simple analytic considerations similar to those used by Khang et al. in their 2D study. We analyze the probabilities of failure around the likely largest cluster of breaks just ahead of the main crack expected at any applied force. The analysis is quantitatively accurate for weak disorder where the damage prior to failure is limited.

The brittle behavior at small $W$ (and observed for $W=0.25$ ) can be understood quite well because it is controlled by a simple defect configuration. For a sufficiently wide crack (large $L$ ), the weakest heterogeneity in the row in front of the crack has a strength $s=1-W / 2$ and so fails at a normalized local (crack tip) force $F=1-W / 2$. The two lateral neighboring sites then experience an increased force $\left(1+\eta_{1}\right) F$, where $\eta_{1}$ is a stress concentration factor $\left(\eta_{1}\right.$ $=0.11$ for the cubic lattice with $E / \mu=1.0$ ). The weaker of the two neighbors has a typical strength of $1-W / 6$ and hence fails immediately if $(1-W / 2)\left(1+\eta_{1}\right)>(1-W / 6)$. Failure of this second spring along the crack front then generally precipitates unstable continued growth of the initial "kink" into a "ledge," followed by complete failure along the width. Rearranging the above inequality, we find that a perfectly brittle regime of failure is expected for

$$
W<3 \eta_{1} /\left(1+1.5 \eta_{1}\right)=0.285
$$

and the measured strength is $F=1-W / 2$. This result is in excellent agreement with the observed brittle behavior at $W$ $=0.25$.

For values of $W$ above the perfectly brittle limit, the very first break along the crack front does not cause immediate failure. Some finite damage occurs prior to the lateral instability. To understand the strength versus $W$ in this regime, consider applying a force $F$ and failing the fraction $p=[F$
TABLE I. Predicted maximum ledge length $c_{\max }$ and associated failure strength $F$ as a function of disorder width $W$ and crack width $L$. Also shown is the minimum force $F_{\text {crit }}$ needed to grow a crack past the toughest heterogeneity encountered along the crack front; when $F<F_{\text {crit }}$, crack extension further ahead of the crack must occur prior to full crack advance.

\begin{tabular}{ccccc}
\hline \hline$W$ & $L$ & $c_{\max }$ & $F$ & $F_{\text {crit }}$ \\
\hline \multirow{2}{*}{0.4} & 40 & 1.5 & 0.84 & 0.77 \\
& 100 & 1.6 & 0.83 & \\
0.5 & 40 & 2.0 & 0.85 & 0.80 \\
& 100 & 2.3 & 0.83 & \\
0.75 & 40 & 2.7 & 0.90 & 0.88 \\
& 100 & 3.5 & 0.88 & \\
1.00 & 40 & 3.2 & 0.97 & 0.96 \\
& 100 & 4.2 & 0.94 & \\
1.50 & 40 & 3.5 & 1.11 & 1.12 \\
& 100 & 5.1 & 1.09 & \\
2.00 & 40 & 3.7 & 1.27 & 1.28 \\
& 100 & 5.6 & 1.24 & \\
& 200 & 6.9 & 1.22 & \\
\hline \hline
\end{tabular}

$-(1-W / 2)] / W$ of sites along the crack front that are weaker than $F$. The number of ledges of length $c$ along the width $L$ is then

$$
N(c)=L(1-p)^{2} p^{c} .
$$

The largest ledge $c_{\max }$ in the width $L$ satisfies

$$
N\left(c_{\max }\right)=1
$$

and the two neighboring lateral springs then have stress concentrations of at least $\eta_{c_{\max }}$. The weaker of the two neighboring springs, taking into account that these surviving sites must be stronger than $F$, has a typical strength of

$$
s=F+[(1+W / 2)-F] / 3 .
$$

The weaker neighbor will then fail, on average, when

$$
s=F\left(1+\eta_{c_{\max }}\right)>F+[(1+W / 2)-F] / 3 .
$$

If this failure event is presumed to lead to unstable lateral growth, then the measured strength is the minimum value of $F$ that satisfies these conditions. Solving Eqs. (5)-(8), using the stress concentration factors $\eta_{c} \approx 0.25-0.14 / c^{0.7}$ measured for this lattice and $E / \mu$ value, yields predicted strengths $F$ versus $W$ and $L$ as shown in Table I. The results agree well with the simulations at smaller $W$, and are consistent with the trend that $F$ increases with increasing $W$ for $W>0.5$. There is also a predicted slow decrease in strength with increasing width $L$, which cannot be clearly observed in the simulations.

The above argument is an underestimate of the failure stress if damage is confined to the row of springs immediately ahead of the crack front because the ledge stress concentration $\eta_{c}$ approaches a maximum value, rather than growing unbounded. Hence the presumed instability will not always lead to complete failure across the entire width. In the extreme case, consider the impingement of two long ledges with a lone site remaining in between. That site ex- 
periences a stress concentration of $\eta=0.557$ and hence will definitely fail, completing the advance of the crack, only if $F(1+0.557)<1+W / 2$. If the $F$ estimated from the analysis of Eqs. (5)-(8) above is less than this value of $F_{\text {crit }}=(1$ $+W / 2) / 1.557$ then the unstable failure assumption used above cannot hold. The value of $F_{\text {crit }}$ is also shown in Table I, and a comparison with the strength estimate from Eqs. (5)-(8) shows that the "imperfect" brittle regime persists up to about $W=1.0$.

For $W \geqslant 1.0$, failure simply cannot occur without some evolution of the crack beyond the first row of springs prior to failure. Such further advance of the crack generates increased stress concentrations along the lagging crack front and tends to drive failure. However, the increased stress concentrations associated with advance of the crack are not, apparently, sufficient to significantly reduce the strength below the simple predicted value. One reason for this is that the diffuse growth of the crack redistributes stress predominantly ahead of the advancing crack (this is the reason that the stress concentration factor at the edge of a ledge reaches a finite maximum). A qualitative argument for the behavior in the strong disorder regime is as follows. At a given applied $F$, the specific distribution of spring strengths $P(s)$ establishes an amplitude of crack advance ahead of the initial crack and a typical correlation length along the width of the crack (e.g., a sine wave front with amplitude $\Delta c$ and lateral wavelength $\lambda$ ). The amplitude establishes a typical stress concentration factor at the lagging, or pinning, sites. The pinning sites are, by definition, strong enough to withstand this stress concentration and the crack is stable. Increasing the applied stress further allows the crack to break through the weaker pinning sites, thereby increasing the wavelength/ correlation length along the crack front but not increasing the amplitude appreciably. This advance only weakly increases the stress concentrations on the remaining pinning sites. The remaining pinning sites generally remain intact, and thus further applied loads are required to induce further depinning and crack advance.

The consequences of the above qualitative picture are several. Being controlled by the stronger pinning sites, a wider crack (larger $L$ ) allows for more strong pinning sites and correspondingly possibly larger toughness. Moreover, a wider crack, viewed as a series of narrower subsections, does not completely advance due to failure of one or a few weak subsections-it is still pinned by the stronger subsections. The crack advance thus follows a strongest-link behavior wherein growth is controlled by the "strong" sections along the front. This "strongest-link" behavior is consistent with the observation that the toughness distribution narrows and shifts slightly upward with increasing width by elimination of the weaker lower tail of the distribution, as evidenced in Fig. 2. It is also consistent with the observation that the total damage $N_{b} / L$ prior to failure has a nontrivial and increasing scaling with the crack width. These "strongest-link" features are in contrast to the weak-link-driven phenomenon, and decreasing damage with increasing volume, usually observed in weakly disordered brittle systems.

\section{DISCUSSION}

For a perfect homogeneous material $(W=0)$ we of course do obtain the thermodynamic toughness, which is larger than in any disordered materials (see Fig. 3). However, any real material will have initial distributed cracklike defects. Even in an otherwise homogeneous material $(W=0)$, these defects will provide the "kinks" along the crack front necessary to drive brittlelike failure at reduced stresses, roughly $F=(1$ $\left.+\eta_{1}\right)^{-1}=0.90$ in the present model. We thus expect reduced toughnesses for all weakly heterogeneous materials $(W \leqslant 0.5)$. Conversely, the highly disordered material is much less sensitive to such defects: the tougher heterogeneities can efficiently pin many various configurations of the crack front. To explicitly demonstrate the differing sensitivities to defects, we have studied crack growth in materials with a small $2.5 \%$ volume fraction of initial randomly distributed breaks ("cracks", or "porosity"). For the homogeneous material $W=0$, the average toughness is measured to be 0.905 as compared to the thermodynamic toughness $\bar{K}=0.975$, which arises because a fraction 0.025 of sites have zero strength. So for small $W$, toughness is controlled by the preexisting defects which form ready-made kinks at the crack front and precipitate failure below the average toughness. For $W=2.0$, the measured average normalized toughness is 0.98 , which is essentially identical to the average normalized thermodynamic toughness of 0.975 , so that the toughness remains only slightly below the average thermodynamic toughness. Hence, there is roughly a $25 \%$ strength difference and $10 \%$ toughness difference between all weakly $(W<0.5)$ and strongly $(W \approx 2)$ disordered materials.

In light of the present results showing small toughness differences between weak and strong disorder, the role of complexity in enhancing toughness in ceramic microstructures must go beyond purely modest heterogeneity effects. Mechanisms such as grain bridging or controlled microcracking are necessary to provide enhanced toughness. Grain bridging occurs in polycrystals when some grains do not fail as the crack passes completely around them. ${ }^{13}$ The bridging grains remain in the wake of the crack front and exert closure forces on the crack faces that counteract the applied forces and lead to enhanced macroscopic toughness. Crack bridging can be viewed, within the context of the present model, as a particular case of a disordered material in which most of the grains have the average toughness while the bridging grains have a much larger effective toughness (effective because of the role played by interface debonding, for example). The larger toughness is sufficiently large that the crack front bypasses the bridging grains and leaves them intact for some distance behind the crack front. Toughening by this "very strong disorder' mechanism is significant, both in real polycrystalline materials ${ }^{13}$ and in models using the same discrete elastic representation of the microstructure employed here. ${ }^{14}$ The toughening studied here, where the local toughnesses vary over a limited range and no bridging phenomenon arises, may be operating in grain-bridged material but its effects may be masked by the larger toughening imparted by the grain bridging. However, disorder can also play a key role in the formation and efficacy of such bridges. This is most clearly evident in fiber-reinforced ceramics where fiber breaks occur out of the plane of the crack, driven by the stochastic distribution of fiber strengths, and create fiber " "bridges", which resist further crack growth. We have recently modeled toughening in such fiber-reinforced materials using similar Green's-function models. ${ }^{12,15}$ As the distribu- 
tion in fiber strengths becomes broader, the fiber bridging stresses become larger because fiber breaks occur further from the crack plane. Furthermore, these bridging stresses are the only source of enhanced toughening and hence higher toughness is associated with greater spread in the fiber strength distribution. ${ }^{16}$ In keeping with the results here, the distribution of fiber strengths in the actual crack plane does not contribute to the toughening of the material to any appreciable extent. These results also indicate that inclusion of crack growth or damage out of plane can make contributions to toughening in systems with elongated microstructures.

The present work does demonstrate the general importance of geometry and dimensionality in modeling crack growth problems. In a 2D system with a $1 \mathrm{D}$ crack, a tough heterogeneity completely impedes crack growth. The measured toughness follows precisely the high-toughness region of the toughness distribution and leads to a prediction of toughness much higher than the average. In a 3D system with a planar crack, the crack can grow around individual tough elements and ultimately overcome the high toughnesses by a correspondingly high stress concentration factor but at lower macroscopic applied loads. The measured toughness is much lower than in $2 \mathrm{D}$, barely attaining the thermodynamic average, although we find that the tougher regions do pin the crack and control crack growth to some extent. Our results show that misleading and perhaps overly optimistic results can be obtained by studying problems in lower dimensions.

In summary, we have explicitly demonstrated that mate- rial heterogeneity affects material toughness in nonintuitive ways. First, toughness is not a pure material parameter and is a statistical quantity. Second, two regimes of behavior exist: weak disorder having toughness below the average thermodynamic value and having planar crack fronts; and strong disorder with toughness approaching the thermodynamic value and a rough crack front. Third, planar cracks in three dimensions evolve in a unique manner and cannot be studied approximately by linear cracks in two dimensions. Fourth, and most important, the magnitude of the toughness difference is not large, so that heterogeneous or distributed toughnesses do not provide an effective toughening mechanism. Future work using the present model will be aimed at (i) mesh refinement in which more detailed microstructure is overlayed onto the spring network, (ii) specific studies of polycrystalline metal and intermetallic fracture, for which realistic grain-boundary distributions and toughnesses are now being established, and (iii) investigation of timedependent degradation of disordered materials, where the sensitivity of damage rates to local stress intensities may have large effects on the magnitudes and distributions of lifetimes of heterogeneous materials.

\section{ACKNOWLEDGMENTS}

The author thanks the National Science Foundation, Division of Materials Research (Materials Theory) for support of this work under Grant No. DMR-9420831, and Dr. Robb Thomson and Professor Z. Suo for illuminating discussions.
${ }^{1}$ B. Lawn, Fracture of Brittle Solids, 2nd ed. (Cambridge University Press, Cambridge, England, 1993); D. R. Clarke and K. T. Faber, J. Chem. Phys. Solids 48, 1115 (1987).

${ }^{2}$ R. W. Rice, S. W. Freiman, and J. J. Mecholsky, J. Am. Ceram. Soc. 63, 129 (1980).

${ }^{3}$ A. G. Evans, Philos. Mag. 26, 1327 (1972); L. R. F. Rose, Mech. Mater. 6, 11 (1987).

${ }^{4}$ A. F. Bower and M. Ortiz, J. Mech. Phys. Solids 38, 443 (1990); 39, 815 (1991); J. R. Rice, J. Appl. Mech. 52, 571 (1985).

${ }^{5}$ B. Khang, G. G. Batrouni, S. Redner, L. deArcangelis, and H. J. Herrmann, Phys. Rev. B 37, 7625 (1988).

${ }^{6}$ M. Sahimi and J. D. Goddard, Phys. Rev. Lett. 33, 7848 (1986); P. M. Duxbury, P. L. Leath, and P. D. Beale, Phys. Rev. B 36, 367 (1987); L. deArcangelis, H. J. Herrmann, A. Hansen, and S. Roux, ibid. 40, 877 (1989); W. A. Curtin and H. Scher, J. Mater. Res. 5, 535 (1990); P. M. Duxbury and P. L. Leath, Phys. Rev. Lett. 72, 2805 (1994); P. L. Leath and P. M. Duxbury, Phys. Rev. B 49, 14905 (1994).

${ }^{7}$ A 1D model has been studied by E. Smith, Mech. Mater. 9, 129 (1990); work similar to the present work is also being carried out by E. Fuller et al. (private communication).

${ }^{8}$ Recent work on 3D systems has been presented by P. M. Duxbury, P. D. Beale, and C. Moukarzel, Phys. Rev. B 51, 3476 (1995), but toughness was not investigated.

${ }^{9}$ H. B. Rosenstock and G. F. Newell, J. Chem. Phys. 21, 1607 (1953).
${ }^{10}$ V. K. Tewary, Adv. Phys. 22, 757 (1973); C. Hsieh and R. Thomson, J. Appl. Phys. 44, 1051 (1973); R. Thomson, V. K. Tewary, and K. Masuda-Jindo, J. Mater. Res. 2, 619 (1988).

${ }^{11} \mathrm{~A}$ huge advantage of this technique is that the solution for the stresses (forces) in all of the springs in the $y=0$ plane requires the inversion of a matrix that is only of a size equal to the number of broken springs, rather than requiring the inversion of a matrix of a size equal to the entire system size (which in this case is actually infinite). Furthermore, for the present problem the system always has a crack of at least length $c$ and width $L$, and the Green's functions in the presence of the initial crack can be calculated once initially and subsequent calculations only involve matrices of a size equal to the extent of crack growth. These aspects make possible an otherwise intractable numerical problem, and allow for the necessary computation of many steps of crack growth.

${ }^{12}$ S. J. Zhou and W. A. Curtin, Acta Met. 43, 3093 (1995).

${ }^{13}$ R. Steinbrech, R. Khehands, and W. Schaarwachter, J. Mater. Sci. 18, 265 (1983); P. L. Swanson, C. J. Fairbanks, B. R. Lawn, and Y. W. Mai, J. Am. Cer. Soc. 70, 279 (1987); S. T. Bennison and B. R. Lawn, Acta Met. 37, 2659 (1989); R. Cook, ibid. 38, 1083 (1990); G. Vekinis, M. F. Ashby, and P. W. R. Beaumont, ibid. 38, 1151 (1990).

${ }^{14}$ W. A. Curtin, J. Am. Cer. Soc. 78, 1313 (1995).

${ }^{15}$ M. Ibnabdelijalil and W. A. Curtin (unpublished).

${ }^{16}$ M. Ibnabdelijalil and W. A. Curtin (unpublished). 